\title{
Study of the Neural Basis for Subjective Feature Binding
}

\author{
Tzu-Ching Chiang ${ }^{1}$, Jyh-Horing Chen ${ }^{2}$, Keng-Chen Liang ${ }^{3}$, Chung-Ping Cheng ${ }^{4}$, Sigmund Hsiao $^{1}$, \\ Chao-Hsien Hsieh ${ }^{2}$, Yun-An Huang ${ }^{2}$, Chia-Wei $\mathbf{L i}^{2}$ \\ ${ }^{1}$ Department of Psychology, National Chung Cheng University, Chia-Yi County, Taiwan \\ ${ }^{2}$ Electrical Engineering, Interdisciplinary MRI Laboratory, National Taiwan University, Taipei, Taiwan \\ ${ }^{3}$ Department of Psychology, National Taiwan University, Taipei, Taiwan \\ ${ }^{4}$ Department of Psychology, National Cheng Kung University, Tainan, Taiwan \\ Email: psytcc@ccu.edu.tw
}

Received November 22, 2012; revised December 24, 2012; accepted January 3, 2013

\begin{abstract}
While it is known that the brain perceives color and motion asynchronously, the specific locations in which the brain binds signals remain unknown. This study distinguishes subjective perception of the capability to bind features and the objective accuracy in feature binding. The stimuli were the same for individual subjects, consisting of random dots (red and green, or yellow and blue) moving either vertically or horizontally. Subjects responded to questions regarding the color or the direction of motion of the dots (objective judgment) and rated their capability in performing the task (subjective judgment). The imaging results of contrasting subjective judgment showed that the activation of the anterior rostral cingulate cortex (rACC) and inferior frontal gyrus (Brodmann area [BA] 45/47) during incapable-of-binding responses, compared with the capable-of-binding responses. It is suggested that the rACC is for uncertainty of subjective judgment and BA $45 / 47$ is for the increased burden on working memory. In contrast, there was no imaging results of contrasting the correct and incorrect responses (i.e., objective judgment), and neither was there for the interaction between subjective and objective judgment. The results of conservative conjunction analysis indicated common and shared brain areas for the 2 distinctive binding situations (the correct and capable-of-binding vs the incorrect and incapable-of-binding), including increased activity in the intraparietal lobe (IPL) and the junction areas of the posterior rostral ACC (dACC) and the prefrontal areas, but decreased activity in the medial portion of the IPL, suggesting that feature binding requires maintaining attention. These results clearly isolated subjective judgment from objective judgment and support the view that maintaining attention is involved in feature binding of color and motion.
\end{abstract}

Keywords: Anterior Cingulate Cortex; Feature Binding; Functional Magnetic Resonance Imaging; Intraparietal Lobe; Inferior Frontal Gyrus

\section{Introduction}

In the realm of visual perception, the features of color and motion are not only processed in separate locations in the cortex [1,2], but are also perceived asynchronously [3], with the conscious perception of color preceding that of motion by 80 to $100 \mathrm{~ms}$. This segregation naturally leads to consideration of the so-called "feature binding problem", that is, determination of how perceptions of color and motion are subsequently recombined to provide a holistic representation of an object within a perceptual domain in which all attributes appear integrated.

There are several kinds of binding issues such as spatial/location, temporal feature, and property binding [4]. This study focuses on the property binding describing several features of an object that share the same spatial location at any time, in order to characterize the object. For example, the binding of color, shape, and motion can be used to characterize a car. There are several general factors that influence property binding. Previous studies have reported that attention is involved in the feature binding mechanism [5-11]. In addition to the attentional factors, understanding the feature-binding mechanism requires considering the subjective nature of the perception of feature binding, which is illustrated by focusing on perceptual integration under awareness [12-14]. To accomplish this, the present study presents constant stimuli while manipulating 2 factors, the capability of binding (subjective judgment of binding performance) and the correctness of the binding (objective judgment of accuracy) (Table 1). The two-by-two factorial design follows experimental designs commonly used in psychological studies, which allows subjective judgment to be distinguished from objective judgment. Furthermore, the use of constant stimuli allows the results to be attributed to the functioning of cortical mechanisms rather than to exposure to external stimuli. The goal of this investiga- 
tion is to provide greater elucidation and understanding of the differences between subjective and objective judgment within the feature binding mechanism.

\section{Methods}

\subsection{Experimental Setup and Stimuli}

While wearing functional magnetic resonance imaging (fMRI)-compatible goggles $\left(14.25^{\circ} \times 10.71^{\circ}\right.$ in width and height, VisuaStim XGA; Resonance Technology Inc., Northridge, California, USA), the 21 study participants were placed in an fMRI scanner and exposed to visual stimuli. The stimuli consisted of either 50 green and 50 red dots or 50 yellow and 50 blue dots on a black background (Figure 1). Half of the dots of each color moved in opposite directions along either a horizontal or vertical axis at a speed of $4.30^{\circ} \mathrm{s}$, not in a straight line but at variable angles within a range of $0.14^{\circ}$ perpendicular to the original movement direction. The other half of the dots of each color flashed at random locations in order to increase task difficulty. Equiluminance of different colors was separately established for each subject by flicker photometry [15] before testing. All visual stimuli were constructed using COGENT Graphics (available at www.vislab.ucl.ac.uk) run in MATLAB (Mathworks
Inc.).

The subjects were instructed that their task was to identify which color was moving in which direction, using a keypad to answer 1 of 2 questions that randomly appeared after presentation of the stimulus. One question asked, "Which color of dots was moving direction?" with the word direction replaced by up, down, left, or right, as appropriate, and one question asked, "In which direction were the color dots moving?" with the word color replaced by green, red, yellow, or blue, as appropriate. The questions were relevant to the stimuli. For instance, if the subjects were shown red and green dots, they were not asked about the directions of blue or yellow dots but that of red and green dots. If they were shown dots moving only horizontally, they were not asked the color of the dots moving vertically but horizontally either to the left or right. The combination of stimuli and the related questions were balanced in a pseudorandom sequence, such that each stimulus type was followed by each of the possible questions in turn within a block of 32 trials.

Each subject's response was objectively categorized as either correct or incorrect, based on accuracy. In addition, each subject subjectively judged himself/herself either capable or incapable of binding the features. The sub ject's judgment was a binary response, with "capable" indicat-

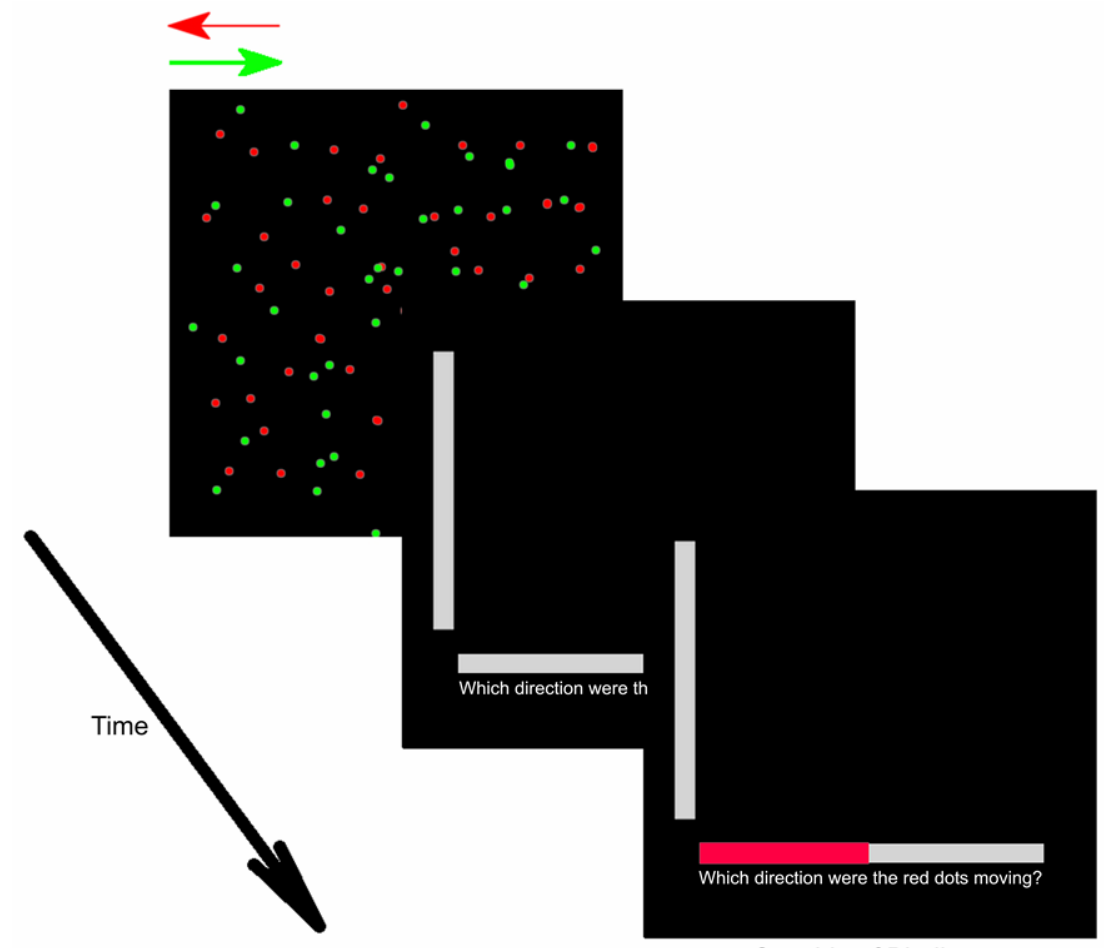

Capable of Binding

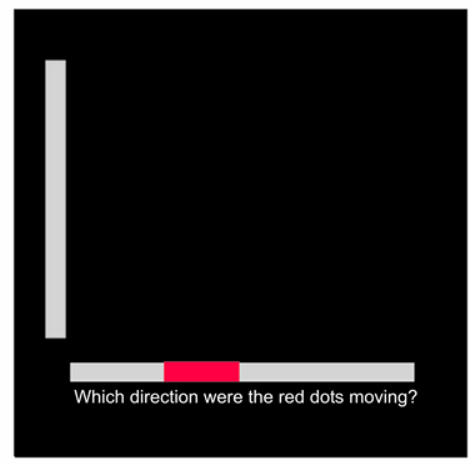

Incapable of Binding

Figure 1. Schematic diagram depicting the study task. The examples shown here are the stimuli consisting of red and green dots moving horizontally for a specific duration. A question then appeared to which the subject responded using the keypad and the response immediately showed up on the relevant grey bar. The question shown here was related to the direction of movement of the green dots, and the subject could provide the same response in the capable-of-binding (on the bottom left) or incapable-of-binding (on the bottom right) condition. 
ing the subject felt that s/he could bind features successfully and "incapable" indicating the subject felt that $\mathrm{s} / \mathrm{he}$ could not do it. No further questions were asked for the subjective assessment. Instead, subjects answered the color/motion question and automatically marked their answer as "capable" or "incapable" at the same time.

Scores A, B, C, and D in Table 1 each correspond to the number of times that a subject's responses fell into a particular category. Score A corresponds to the number of times that a subject responded correctly and reported that he or she was able to bind the features; Score B, the number of times that a subject responded correctly, but reported that he or she was unable to bind the features; Score $\mathrm{C}$, the number of times that a subject responded incorrectly, but reported that he or she was able to bind the features; Score D, the number of times that a subject responded incorrectly and reported that he or she was not able to bind the features. The only way to confirm the subjective declaration of capability or incapability of feature binding was to compare the subjective assessment against the objective accuracy data.

If an individual's subjective assessment of their capability of feature binding was accurate, the comparison of their subjective assessment against the objective data would be expected to yield results significantly greater than those obtained by chance alone (i.e., $\mathrm{A} /(\mathrm{A}+\mathrm{C}) \geq$ $68 \%$ ), based on the reasoning that the minimum probability of obtaining a value significantly higher than $50 \%$ is 0.6732 when the $\alpha$-value is set to 0.05 . Setting the threshold of 0.6732 for the positive binding condition did not affect the incapable binding trials (i.e., $\mathrm{B}+\mathrm{D}$ ), in which a $50 \%$ threshold was used, because the accuracy in these trials is expected to be no greater than chance (0.5). These criteria were applied to individual subjects. Subjects whose data did not meet these criteria ( 2 of the 21 subjects) were excluded from the study.

\subsection{Subjects}

Twenty-one healthy subjects (8 males and 13 females) between 19 and 30 years of age (mean \pm SD, $22.7 \pm 2.7$ ) participated in the fMRI experiment. All subjects were

Table 1. Response categories. Responses were categorized according to the assessment of the capability of binding features (subjective judgment) and accuracy (objective judgment) in binding. Capable binding was represented as the combination of Cell $A$ and Cell $C$, if and only if $A /(A+C) \geq$ 0.68 , while incapable binding was represented as the combination of Cell $B$ and Cell $D$, if and only if $B /(B+D) \approx 0.5$.

\begin{tabular}{cccc}
\hline & & \multicolumn{2}{c}{ Capability of Binding } \\
\hline & & Capable & Incapable \\
Response & Correct & $\mathrm{A}$ & $\mathrm{B}$ \\
& Incorrect & $\mathrm{C}$ & $\mathrm{D}$ \\
\hline
\end{tabular}

right-handed and had normal or corrected-to-normal vision. Written informed consent was obtained from all subjects. The study was approved by institutional review board of the Department of Psychology, National Chung Cheng University, Taiwan. Each subject received 1000 Taiwan Dollars as compensation for his/her time and travel costs.

\section{3. fMRI Scanning Methods}

All functional scanning was performed with a 3-T Bruker 30/90 Medspec fMRI scanner fitted with a standard birdcage head coil (Bruker BioSpin MRI GmbH; Ettlingen, Germany). An echo-planar imaging (EPI) sequence was applied for functional scans measuring blood oxygen level dependent (BOLD) signals (echo time (TE) = $30 \mathrm{~ms}$; repeat time $(\mathrm{TR})=3$ seconds). Each brain image was acquired in an interleaved sequence from the bottom of the brain to the top, comprising 80 volumes of 35 axial slices; each slice was $3.75-\mathrm{mm}$ thick with no gap between the slices, had a resolution of $3.75 \times 3.75 \times 3.75 \mathrm{~mm}$, and covered nearly the entire brain. The first 7 volumes of each scanning session were discarded to allow for T1equilibrium effects. T1-weighted axial anatomical scanning was performed after functional scanning to obtain high-resolution structural images comprising 35 axial slices, each with a resolution of $0.9375 \times 0.9375 \times 3.75$ $\mathrm{mm}$ with no gap between the slices $(\mathrm{TE}=39.4 \mathrm{~ms}, \mathrm{TR}=$ $614.2 \mathrm{~ms}$, flip angle $=90^{\circ}$, field of view [FOV] $=240$ $\mathrm{mm})$.

\subsection{Procedure}

Before initiating functional scanning, the duration of stimulus presentation for each subject was determined using the method of limits. Using this method, the subject was first provided with the longest duration ( 2 seconds) of stimulus presentation with which to perform feature binding, to ensure that the subject was capable of feature binding within this duration. The subject was then provided with succeeding durations each reduced by half until the subject reported that he or she could not bind the features. Next, the subject was provided with the shortest duration $(32 \mathrm{~ms})$ with which to perform feature binding, to ensure that the subject could not perform feature binding within this duration. The subject was then provided with succeeding durations that each increased by two-fold until the subject reported that he or she could now bind the features. The descending and ascending order of duration was repeated 3 times per subject while varying the longest and shortest durations. After completion of this process, a constant duration was chosen and tested for 64 trials ( 2 blocks of 32 trials) to ensure that the subject could meet the accuracy criteria defined above. If a subject failed to meet the performance criteria, an- 
other duration was selected with which to repeat testing until the performance criteria had been fulfilled. The optimal stimulus duration among the subjects ranged from $53.5 \mathrm{~ms}$ to $401.1 \mathrm{~ms}($ mean $=155.5 \mathrm{~ms}, \mathrm{SD}=107.9 \mathrm{~ms})$. The variation of duration did not affect performance. Each subject underwent 2 scanning sessions consisting of a total of 64 trials. Each trial consisted of a single presentation of the visual stimuli for a specific duration, after which a question appeared at the bottom of the screen for a 6 seconds $(=2 \times \mathrm{TR})$ period. During this period, the subjects indicated their response to the question and reported their capability of binding the features of color and motion. The next trial automatically began after $6 \mathrm{~s}$ had passed.

\subsection{Behavioral Data Analysis}

The aim of behavioral data analysis was to confirm that the selected duration for individual subjects was appropriate and to confirm accuracy of the subjective responses. The measurement of accuracy was calculated and categorized according to whether the subject was capable or incapable of performing feature binding. As the accuracy data were used as indices, the response for each trial was considered a sample of binomial data. The non-linear mixed effect (NLMX) regression model was used to analyze the binomial data $[16,17]$. To conduct NLMX analysis, the accuracy of the fMRI experiment was modeled using the following equation:

$$
p=p 0+p 1 * x 1+\varepsilon
$$

where $p$ was the accuracy of either the capable-of-binding or incapable-of-binding trials. Dummy coding was used in the regression model: $x 1=0$ represented incapable-of-binding trials and $x 1=1$ represented capable-ofbinding trials. The error among subjects, $\mathcal{E}$, was assumed to fit the standard normalized distribution. According to the dummy coding, the estimate of $p 0$ was the mean accuracy of the incapable-of-binding trials and the estimate of $p 1$ was the difference in accuracy between the capable-of-binding and incapable-of-binding trials.

\section{6. fMRI Data Analysis}

The fMRI data were first preprocessed using the SPM8 software (Wellcome Trust Centre for Neuroimaging, London, UK, http://www.fil.ion.ucl.ac.uk/spm). Each functional scan of individual subjects was realigned to the average of all volumes obtained (i.e., the 73 volumes that remained after deleting the first 7 volumes for the T1equilibrium effect) without unwarping and was re-sliced for the time correction. Next, each subject's structural image was translated to match the first EPI volume for co-registration between the anatomic and functional scans. The structural image was then segmented into grey and white matter with an East Asian brain as the spatial template for the Affine regularization. The realigned and resliced images were then spatially normalized to the canonical template provided by the SPM8 software and spatially smoothed with a Gaussian kernel of $8 \mathrm{~mm}$ full width at half maximum (FWHM). The pre-processed data were then subjected to first-level analysis using a voxel-wise general linear model (GLM) that included regressors defining stimulus onsets for each of the 4 factorial conditions (see Table 1). The number of button presses and motion correction parameters were treated as effects of non-interest. Appropriate regressors were convolved with the default SPM hemodynamic response function (HRF) with 2 additional derivatives of a time derivative and a spatial dispersion. The default HRF function parameters described the BOLD intensity signals since the onset of stimuli. For example, the length of the kernel was $32 \mathrm{~s}$; the delay of peak BOLD responses was $6 \mathrm{~s}$ relative to onset; the delay of post-stimuli undershoot was $16 \mathrm{~s}$.

Restricted maximum likelihood (ReML) inference was used to estimate the model parameters. The estimated parameters were applied to create contrasts in the factorial design, which were then employed to perform random-effects analysis between subjects (second-level analysis) using one-sample t-testing with classical inference (ReML). The final outcome of these analyses was a group analysis, resulting from the pooling of data across the subjects. The statistical results were based on the uncorrected $\mathrm{p}$-value $(\mathrm{p}=0.001)$ and used to choose clusters (voxel number $\geq 5$ in a cluster) that passed the criterion of multiple comparisons with a family-wise error (FWE) corrected p-value of 0.05 at the cluster level. The coordinates of all activation sites were based on the reference brain provided by the Montreal Neurological Institute (MNI). Conservative conjunction analysis was performed in the statistical parametric mapping (SPM) module to identify the common and shared areas between distinctive contrasts $[18,19]$, such as Cell A and Cell D in Table 1. Two-sample t-testing coupled with classical inference (ReML) estimation was performed at the 2nd level of analysis to conduct group conjunction analysis. The results were illustrated with an xjView toolbox (http://www.alivelearn.net/xjview).

\section{Results}

Based on the duration selection procedure described above, the average number of trials in Cells A, B, C, and D were 40.2 (SD 6.7), 5.7 (SD 3.4), 10.2 (SD 4.3), and 7.7 (SD $4.7)$, respectively. The unbalanced number of trials $\left(X_{1}^{2}\right.$ $=7.08, \mathrm{p}<0.01)$ was the result of $>70 \%$ accuracy in the capable-of-binding trials and only 50\% accuracy for incapable-of-binding trials. Figure 2 shows the mean ac- 
curacy for the capable-of-binding trials (0.7977), which was significantly higher than the accuracy of incapableof-binding trials $(0.4279$; standard error of the 2-group difference $[\mathrm{SED}]=0.03661, \mathrm{t}(18)=-10.10, \mathrm{p}<0.0001)$. These results illustrated that the selection of stimulus duration was appropriate that was driving the accuracy of the subjective determination of feature binding. Furthermore, no significant differences were found among subjects with regard to response distribution $(\mathrm{t}(18)=0.38, \mathrm{p}$ $=0.7070)$, indicating that none of the subjects bound features more or less frequently compared to other subjects.

The results of the second-level analysis of the imaging comparisons are shown in Figure $\mathbf{3}$ and Table 2. The comparison of subjective judgment on the capability of feature binding, (Cell A + Cell C) vs (Cell B + Cell D) (i.e., capable- vs incapable-of-binding), indicated significant deactivation of the anterior rostral cingulate cortex (ACC, corresponding to BA 24/32, FWE $\mathrm{p}<0.05$ at the cluster level) and the inferior frontal gyrus (BA 45/47, FWE $\mathrm{p}<0.05$ at the cluster level). It is worth noting that there were no significant differences for the comparison of objective judgment, (i.e., (Cell A + Cell B) vs (Cell C + Cell D)), and there were no significant differences for the comparison of interaction between subjective and objective judgment. Nevertheless, the results of group conjunction analysis of Cell A and Cell D indicated activation in the left intraparietal lobe (IPL), the junction areas of the posterior rostral ACC, and the prefrontal areas, but deactivation in the left medial portions of the IPL (FWE $\mathrm{p}<0.05$ at the cluster level).

\section{Disucssion}

This study examined the feature binding by using random dots moving, while controlling for the 2 variables of subjective perception and objective accuracy. Based on the results of the examination, the subjects' responses were categorized into one of the combination of the two variables (Cells A, B, C, and D). In theory, the 2 variables assessed were not orthogonal because the number of correct responses was proportional to the capability of binding (i.e., a higher capability resulted in a higher number of correct responses). The bias is unavoidable due to the inherent reliability of an individual's subjective judgment based on their perception. The statistical analysis used in this study has also taken into account the unbalanced number of cells by including the observation number (n) in the formula for the t-test. Results using individuals' subjective judgment indicated deactivation in the anterior rostral parts of the ACC (BA 24/32) and inferior frontal gyrus (BA 45/47) when subjects declared that they were incapable of binding features, compared with the capable-of-binding responses. Furthermore, con-

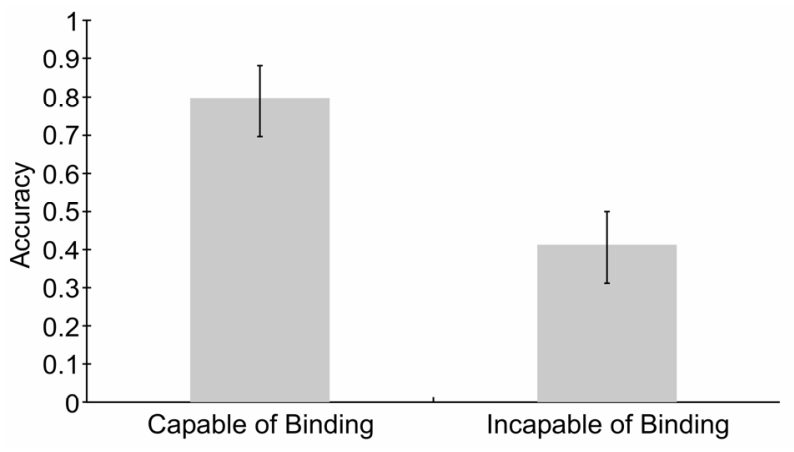

Figure 2. Distribution of accuracy of the fMRI data grouped by the capability of performing binding (see the Methods section for details). Using the non-linear mixed effect model (NLMX), the accuracy of the capable-of-binding responses was 0.7977 (SD 0.085 ) and that of the incapableof-binding responses 0.4279 (SD 0.100 ), a difference that is statistically significant $(t(18)=-10.10, p<0.0001)$. Error bars indicated standard deviation.

junction analysis of Cell A and Cell D was performed to identify the mutually activated areas when performing the binding process and the results indicated activation of the posterior rostral parts of the ACC and IPL and deactivation of the medial parts of the IPL.

Although the initial positions of the random dots presented to the subjects differed in each trial, the same stimuli were presented. Thus, the fMRI results cannot be attributed to stimulus factors but rather to the activation of brain areas corresponding to operations/processing. According to the zero-correlation analysis of unconscious knowledge [20], the fact that subjects who believed they were incapable of binding responded with an accuracy no better than the level of chance ( $\sim 50 \%$ correct) can be interpreted to mean that these subjects had no knowledge of the binding. In fact, there are 2 possibilities: either the subject could not integrate color and motion, or the subject correctly integrated the features but was unaware of doing so. If the latter case occurred frequently, the ratio of correct responses in the incapable-of-binding trials should have been $>50 \%$, as unconsciously integrated perception may still have influenced subjective responses. Such a phenomenon has been noted in cases of blind sight and other conditions in which subliminally perceived stimuli have a detectable influence upon activated brain areas $[21,22]$. However, the accuracy of the incapable-of-binding responses in this study does not support this prediction. Furthermore, no feedback was provided to the subjects during the experiment. As a result, subjects had no knowledge regarding the accuracy of each response. Therefore, we assumed that the declaration of "incapable" meant they were not confident in their ability to successfully bind the features and, similarly, the declaration of "capable" meant they were confident in their ability to successfully bind the features. 


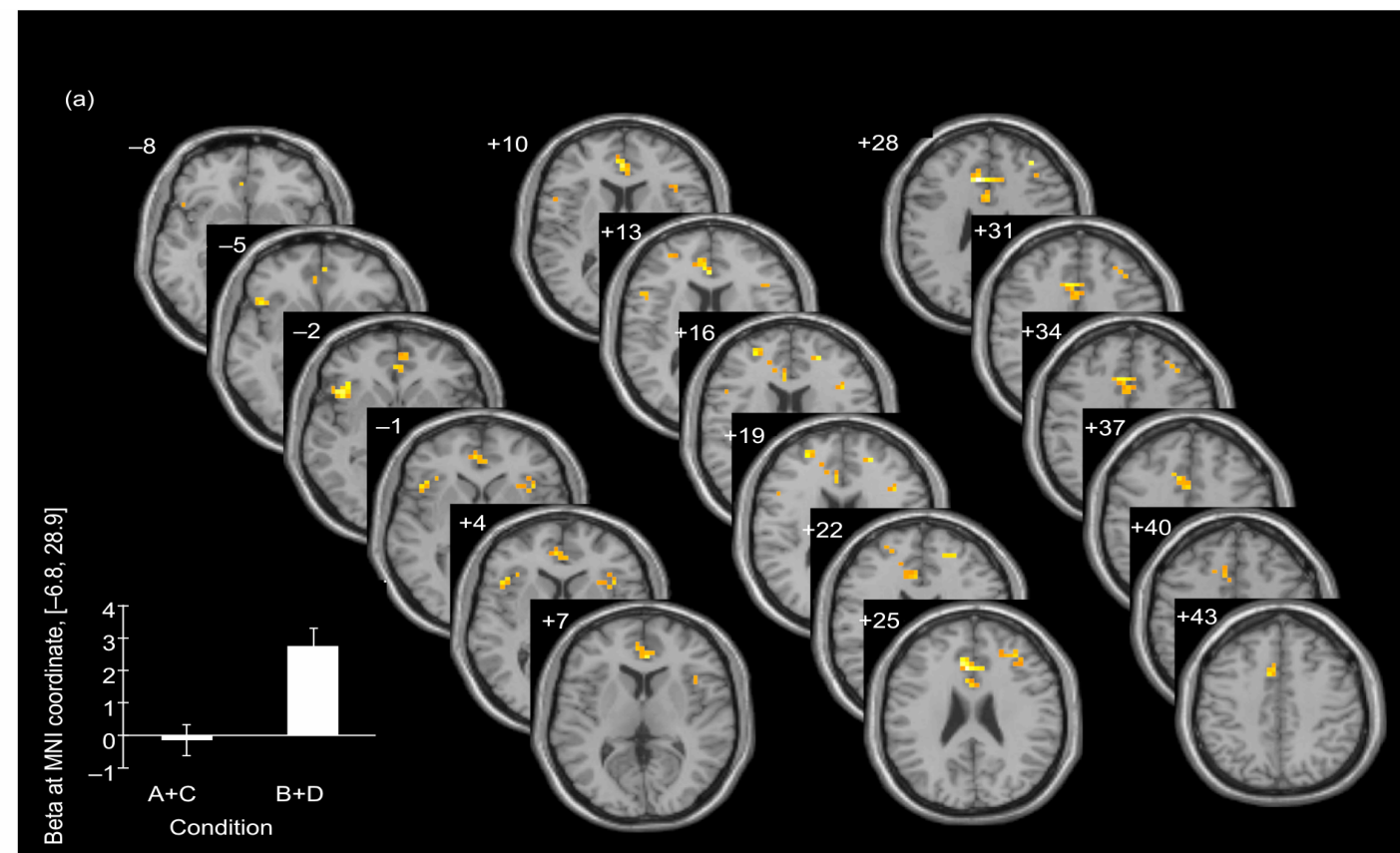

(b)
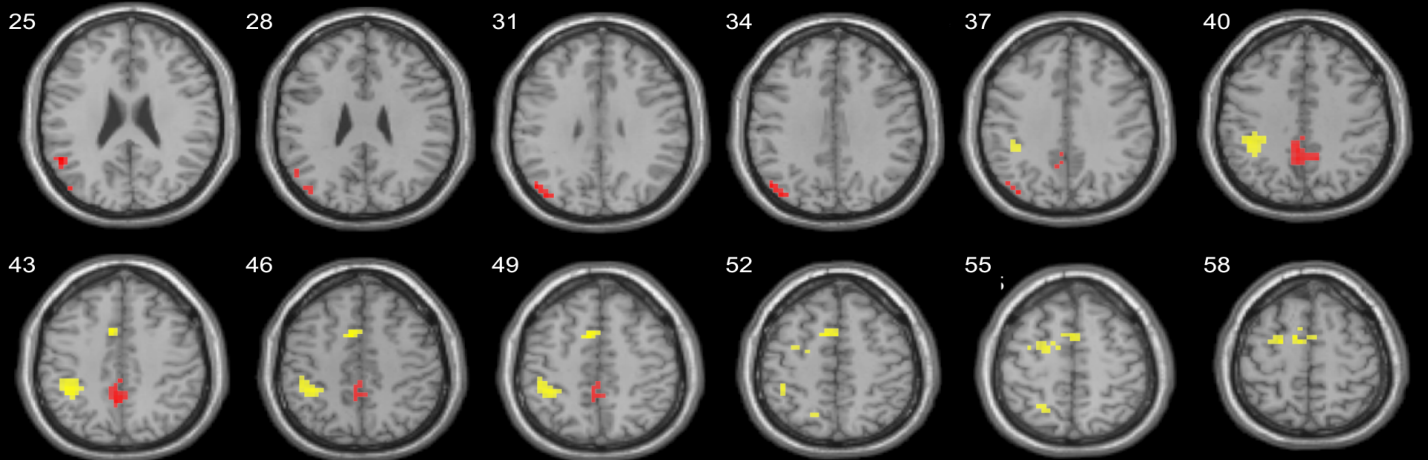

58
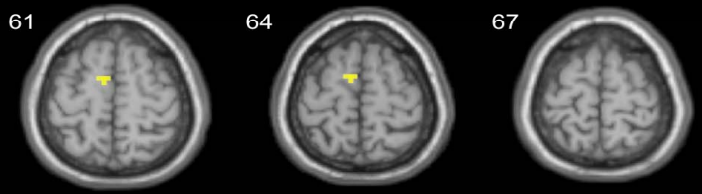

Figure 3. Imaging results. (a) Analysis of the statistical parametric mapping (SPM) results of the comparison of subjective judgment, capable-of-binding vs incapable-of-binding (i.e., Cell A + Cell C vs Cell B + Cell D), indicating deactivation in the anterior rostral cingulate cortex ( $\mathrm{AACC}$ ) and inferior frontal gyrus (BA 45/47). An uncorrected threshold of $p=0.001$ was used for the illustration and the SPM results (FWE p-value $<0.05$ ) were superimposed onto the SPM toolbox XjView, with a template structural brain indexed for the coordinate of the z-axis, according to the Montreal Neurological Institute (MNI) space. Inset shows the average beta values of the comparison across subjects. Error bars represent the standard errors of the average beta values. (b) Conservative conjunction analysis of Cell A and Cell D. A SPM with an FWE p-value of 0.05 at the cluster level indicates increased canonical BOLD responses (in yellow) in the left intraparietal lobe (IPL) and at the junction of the ACC and prefrontal areas, and decreased activity (in red) in the medial part of the IPL. The images were illustrated with the uncorrected $p$-value $(p=0.0001)$ before being superimposed onto the SPM toolbox using XjView, with a template structure brain in a horizontal slice view, and indexed for the coordinate of the $z$-axis, according to the MNI space. 
Table 2. Brain areas identified by the results of imaging comparisons. Two comparisons were statistically significant, 1 of (Cell A + Cell C) vs (Cell B + Cell D) (i.e., subjective judgment) and 1 of the conjunction of Cell A and Cell D. All comparisons were used in 1-sample t-testing at the 2 nd-level of SPM with a FWE of $p<0.05$ at the cluster levels. Positive T values indicate activation of comparisons and negative $T$ values indicate deactivation of comparisons. "Abbreviations: BA 24/32: Brodmann areas 24 and 32; BA 45/47: Brodmann areas 45 and 47; IPL: intraparietal lobe; MNI: Montreal Neurological Institute.

\begin{tabular}{|c|c|c|c|c|c|c|}
\hline \multirow{2}{*}{ Contrast } & \multirow{2}{*}{ Location } & \multirow{2}{*}{ No. of voxels in a cluster } & \multirow{2}{*}{ Peak T value } & \multicolumn{3}{|c|}{ Peak MNI coordinate } \\
\hline & & & & $\mathrm{x}$ & $\mathrm{y}$ & $\mathrm{z}$ \\
\hline \multirow{2}{*}{$\begin{array}{c}(\text { Cell A + Cell C) vs } \\
\text { (Cell B+ Cell D) }\end{array}$} & Anterior cingulate cortex (BA 24/32) & 135 & -7.09 & -7 & 27 & 29 \\
\hline & Inferior frontal gyrus (BA 45/47) & 22 & -5.38 & -41 & 19 & -1 \\
\hline \multirow{3}{*}{$\begin{array}{l}\text { Conjunction of } \\
\text { Cells A and D }\end{array}$} & $\begin{array}{l}\text { Junction of prefrontal cortex } \\
\text { and anterior cingulate cortex }\end{array}$ & 19 & 5.32 & -7 & 8 & 48 \\
\hline & IPL & 32 & 4.9 & -41 & -41 & 44 \\
\hline & Medial parts of IPL & 11 & -4.64 & -3 & -52 & 44 \\
\hline
\end{tabular}

The areas of the ACC involved in the subjective judgment of feature binding are located in the anterior rostral portions (rACC) of the medial frontal cortex (MFC), whereas the dorsal MFC (dACC) is involved in the conjunction analysis of Cell A and Cell D. The rACC may play an evaluative role in monitoring and adjusting the level of control needed for association with the lateral prefrontal cortex, especially under conditions of uncertainty $[23,24]$. Brown and Braver [25] found that the ACC participated in cognitive control over the tracking of a forthcoming event, even in the absence of an error or response conflict, while the findings of a recent imaging study indicated that the ACC and lateral prefrontal cortex are activated during decision-making under conditions of uncertainty [26]. In fact, our imaging results also support the notion that the lateral prefrontal cortex is involved in subjective judgment, although these results approach statistical significance (FWE $p=0.069$ at the cluster level). Thus, in cases of uncertainty, the rACC may help the lateral prefrontal cortex dynamically monitor brain processing and adjust the activation of the lateral prefrontal cortex. This reasoning explains the much higher activity level of the rACC observed in the declaration of incapable-of-binding compared to capable-of-binding responses. Based on this scenario, our results support the role of uncertainty for the rACC and add new information on the subjective judgment of perceptual binding of color and motion.

The inferior frontal gyrus (BA 45/47) is well known for language processing, such as semantic understanding [27-30], action understanding, and the motor mirror system [31-33]. In recent studies, BA 45/47 was also involved in working memory in which the information associated with different features is required to be consistently updated and is bound into a new object $[34,35]$. The increased activation of BA 45/47 in our current study for the incapable-of-binding trials compared to ca- pable-of-binding trials, could therefore be explained by the brain continuing to update the binding of color and motion and the associated increase in online working memory. Another factor increasing the burden on working memory is that the task question in our current study appeared after the end of stimuli presentation. When feature binding failed during the perception stage (i.e., the presentation of stimuli), subjects would make increased efforts to recall the relevant stimulus information, but with poor results.

The possible reasons why no other brain areas were identified in the comparisons of objective judgment (correct vs incorrect binding) are that the brain areas involved are insensitive to this comparison, especially under voxel-wise analysis with SPM. In 2 laboratory studies, Lu et al. [36,37] found that BOLD signals of visual cortices (V1 to V4) depend only on the stimulus contrast being invariant to the general task difficulty. In contrast, their behavior data revealed significant differences between levels of task difficulty. The results of the current study, in which the stimuli remained constant, suggest that binding could occur within brain areas insensitive to task performance. Because some features are coded together in the early visual cortices that respond to multiple dimensions, such as color, motion, and orientation [38], these cortices have been suggested to be the sites of feature binding [39-43]. Another brain area involved in binding could be the pulvinar, which has been observed to play a role in the feature conjunction task [44-46]. Moreover, the pulvinar has clearly been demonstrated to be a part of the cortico-thalamo-cortical loops [47], and has been proposed to be the integrator of visual information and the coordinator of the attentional network in concert with the fronto-parietal network [48]. One potential avenue of further research for binding sites is to use pattern recognition to examine the activity patterns formed by the interested voxels of a specific area, instead of exam- 
ining the activity of individual voxels adopted by SPM.

In order to further explore the binding mechanism and reveal common processing sites, conjunction analysis of Cell A and Cell D was performed. Cell D rather than Cell B + Cell D was selected for analysis because Cell A and Cell B already shared a fixed component, i.e., the correct responses made by subjects. In other words, if conjunction analysis of Cell A and Cell B + Cell D had been performed, the results would have been confounded by the brain areas corresponding to the correct responses. In contrast, Cell A and Cell D contained the different components of correctness and capability of binding. The results indicated activation of the lateral parts of the intraparietal lobe (IPL) and deactivation of the medial parts of the IPL, a finding compatible with the previous finding that the parietal lobe is necessary for feature binding $[6,8$, 10]. However, different parts of the parietal lobe assume different roles in visual attention. The intraparietal sulcus (IPS) has been clearly demonstrated to exhibit enhanced activities during the voluntary and stimulus-driven shifts of spatial attention [49-52], while the superior parietal lobe (SPL) has been shown to play a role in maintaining and tracking the current locus of attention, especially in the peripheral visual field [53]. In contrast, the medial area of the parietal lobe has been related to transient shift-related signals [52], as it is domain-independent and is associated with shifts in attention [54,55].

Consideration of these findings, together with the increase in IPL activity observed in this study, suggests that completing the study task (in both Cells A and D) required initiating and maintaining spatial attention in the peripheral visual field. In addition, the decrease in activity observed in the medial part of the IPL suggests that the shifts in attention that occurred during task completion may have been irrelevant and therefore inhibited. It is worth noting that the task did not manipulate the attentional focus on color or motion, as well as the fact that it would have been difficult for the subjects to shift attentional focus between color and motion because the duration of stimulus exposure was relatively brief. According to the Boolean map theory [56-58], if the subjects had adopted a strategy of shifting attention from 1 color to another color or from 1 motion to another motion, they would have had to expend extra time or cost to perform the attentional shift, leaving them with little time for feature binding after attentional shifting and thus leading to binding failure. As the Boolean map of the task contained and simultaneously presented the dimensions of color and its moving direction, the subjects could only attentionally access one Boolean map at a time. The content of the Boolean map is detected/processed in a parallel way. As a result, the color and its moving direction, or the moving direction and its color were within a Boolean map in which the act of detection was so rapid that it did not require the expenditure of extra time or cost. Therefore, the best strategy for successful feature binding is maintaining attention on one Boolean map (i.e., on one color and its moving direction, or one direction in which the dots are moving and their color).

\section{Conclusion}

This study examined the mechanism underlying the binding of color and motion by performing conjunction analysis and comparing subjective and objective judgment using the same stimuli to exclude the possibility of stimulus attributions to imaging results. The imaging results indicate that the rACC and BA 45/47 are negatively correlated with the capable-of-binding responses (Cell A + Cell C) as opposed to incapable-of-binding responses (Cell B + Cell D). This suggests the uncertainty of incapability-to-bind features and also that working memory is involved in feature binding. However, the binding site may not be sensitive to BOLD signals, especially in the visual cortices. The results of conjunction analysis of the 2 binding conditions (Cell A and Cell D) revealed the mutually activated brain areas to be the lateral and medial parts of IPL, suggesting that the maintenance of attention on stimuli is necessary for performing feature binding processing.

\section{Acknowledgements}

We thank the interdisciplinary MRI/MRS laboratory and C.-H. Hsieh and J.-H. Chen of the Instrumentation Centre, National Taiwan University for assistance with the MRI experiments. This work was supported by the National Science Council, Taiwan (NSC-97-2410-H-194112).

\section{REFERENCES}

[1] M. Livingstone and D. Hubel, "Segregation of Form, Color, Movement, and Depth: Anatomy, Physiology, and Perception," Science, Vol. 240, No. 4853, 1988, pp. 740749. doi:10.1126/science. 3283936

[2] S. Zeki, "Functional Specialisation in the Visual Cortex of the Rhesus Monkey," Nature, Vol. 274, No. 5670, 1978, pp. 423-428. doi:10.1038/274423a0

[3] K. Moutoussis and S. Zeki, "A Direct Demonstration of Perceptual Asynchrony in Vision," Proceedings of Biological Sciences, Vol. 264, No. 1380, 1997, pp. 393-399. doi:10.1098/rspb.1997.0056

[4] A. Treisman, "The Binding Problem," Current Opinion in Neurobiology, Vol. 6, No. 2, 1996, pp. 171-178. doi:10.1016/S0959-4388(96)80070-5

[5] E. Ashbridge, V. Walsh and A. Cowey, "Temporal Aspects of Visual Search Studied by Transcranial Magnetic Stimulation," Neuropsychologia, Vol. 35, No. 8, 1997, pp. 1121-1131. doi:10.1016/S0028-3932(97)00003-1 
[6] M. Corbetta, et al., "Superior Parietal Cortex Activation during Spatial Attention Shifts and Visual Feature Conjunction," Science, Vol. 270, No. 5237, 1995, pp. 802805. doi:10.1126/science.270.5237.802

[7] M. Corbetta, C. M. Sylvester and G. L. HShulman, "The Frontoparietal Attention Network," In: The Cognitive Neurosciences, MIT Press, Cambridge, 2009, pp. 219-233.

[8] S. R. Friedman-Hill, L. C. Robertson and A. Treisman, "Parietal Contributions to Visual Feature Binding: Evidence from a Patient with Bilateral Lesions," Science, Vol. 269, No. 5225, 1995, pp. 853-835.

doi:10.1126/science.7638604

[9] S. Kastner, S. A. McMains and D. Beck, "Mechanisms of Selective Attention in the Human Visual System: Evidence from Neuroimaging," In: The Cognitive Neurosciences, MIT Press, Cambridge, 2009, pp. 205-217.

[10] M. Oliveri, et al., "Facilitation of Bottom-Up Feature Detection Following rTMS-Interference of the Right Parietal Cortex," Neuropsychologia, Vol. 48, No. 4, 2010, pp. 1003-1010. doi:10.1016/j.neuropsychologia.2009.11.024

[11] A. Treisman and G. Gelade, "A Feature-Integration Theory of Attention," Cognitive Psychology, Vol. 12, No. 1, 1980, pp. 97-136. doi:10.1016/0010-0285(80)90005-5

[12] S. M. Fleming and R. J. Dolan, "The Neural Basis of Metacognitive Ability," Philosophical Transactions of the Royal Society B-Biological Sciences, Vol. 367, No. 1594, 2012, pp. 1338-1349. doi:10.1098/rstb.2011.0417

[13] H. C. Lau and R. E. Passingham, "Relative Blindsight in Normal Observers and the Neural Correlate of Visual Consciousness," Proceedings of the National Academy of Sciences, Vol. 103, No. 49, 2006, pp. 18763-18768. doi:10.1073/pnas.0607716103

[14] D. Rosenthal, "Higher-Order Awareness, Misrepresentation and Function," Philosophical Transactions of the Royal Society B-Biological Sciences, Vol. 367, No. 1594, 2012, pp. 1424-1438. doi:10.1098/rstb.2011.0353

[15] P. K. Kaiser, "Flicker as a Function of Wavelength and Heterochromatic Flicker Photometry," In: Vision and Dysfunction, Basingstoke, MacMilliap, 1991, pp. 171-190.

[16] N. E. Breslow and D. G. Clayton, "Approximate Inference in Generalized Linear Mixed Models," Journal of Computational and Graphical Statistics, Vol. 88, No. 421, 1993, pp. 9-25.

[17] T. F. Jaeger, "Categorical Data Analysis: Away from ANOVAs (Transformation or Not) and towards Logit Mixed Models," Journal of Memory and Language, Vol. 59, No. 4, 2008, pp. 434-446. doi:10.1016/j.jml.2007.11.007

[18] K. J. Friston, W. D. Penny and D. E. Glaser, "Conjunction Revisited," Neuroimage, Vol. 25, No. 3, 2005, pp. 661-667. doi:10.1016/j.neuroimage.2005.01.013

[19] T. Nichols, et al., "Valid Conjunction Inference with the Minimum Statistic," Neuroimage, Vol. 25, No. 3, 2005, pp. 653-660. doi:10.1016/i.neuroimage.2004.12.005

[20] Z. Dienes, "Subjective Measures of Unconscious Knowledge," Progress in Brain Research, Vol. 168, 2007, pp. 49-64.
[21] H. C. Lau and R. E. Passingham, "Unconscious Activation of the Cognitive Control System in the Human Prefrontal Cortex," Journal of Neuroscience, Vol. 27, No. 21, 2007, pp. 5805-5811. doi:10.1523/JNEUROSCI.4335-06.2007

[22] A. Sahraie, et al., "Pattern of Neuronal Activity Associated with Conscious and Unconscious Processing of Visual Signals," Proceedings of the National Academy of Sciences, Vol. 94, No. 17, 1997, pp. 9406-9411. doi:10.1073/pnas.94.17.9406

[23] M. M. Botvinick, "Conflict Monitoring and Decision Making: Reconciling Two Perspectives on Anterior Cingulate Function," Cognitive, Affective, \& Behavioral Neuroscience, Vol. 7, No. 4, 2007, pp. 356-366. doi:10.3758/CABN.7.4.356

[24] M. F. Rushworth and T. E. Behrens, "Choice, Uncertainty and Value in Prefrontal and Cingulate Cortex," Nature Neuroscience, Vol. 11, No. 4, 2008, pp. 389-397. doi:10.1038/nn2066

[25] J. W. Brown and T. S. Braver, "Learned Predictions of Error Likelihood in the Anterior Cingulate Cortex," Science, Vol. 307, No. 5712, 2005, pp. 1118-1121. doi:10.1126/science. 1105783

[26] H. Ohira, et al., "Brain and Autonomic Association Accompanying Stochastic Decision-Making," Neuroimage, Vol. 49, No. 1, 2010, pp. 1024-1037. doi:10.1016/j.neuroimage.2009.07.060

[27] M. H. Davis, et al., "Dissociating Speech Perception and Comprehension at Reduced Levels of Awareness," Proceedings of the National Academy of Sciences, Vol. 104, No. 41, 2007, pp. 16032-16037. doi:10.1073/pnas.0701309104

[28] K. Hoenig and L. Scheef, "Mediotemporal Contributions to Semantic Processing: fMRI Evidence from Ambiguity Processing during Semantic Context Verification," Hippocampus, Vol. 15, No. 5, 2005, pp. 597-609. doi:10.1002/hipo. 20080

[29] J. M. Rodd, M. H. Davis and I. S. Johnsrude, "The Neural Mechanisms of Speech Comprehension: fMRI Studies of Semantic Ambiguity," Cereb Cortex, Vol. 15, No. 8, 2005, pp. 1261-1269. doi:10.1093/cercor/bhi009

[30] M. Z. Zempleni, et al., "Semantic Ambiguity Processing in Sentence Context: Evidence from Event-Related fMRI," Neuroimage, Vol. 34, No. 3, 2007, pp. 1270-1279. doi:10.1016/j.neuroimage.2006.09.048

[31] A. F. Hamilton and S. T. Grafton, "Action Outcomes Are Represented in Human Inferior Frontoparietal Cortex," Cereb Cortex, Vol. 18, No. 5, 2008, pp. 1160-1168. doi: $10.1093 /$ cercor $/$ bhm 150

[32] M. Iacoboni, et al., "Grasping the Intentions of Others with One's Own Mirror Neuron System," PLoS Biology, Vol. 3, No. 3, 2005, p. e79. doi:10.1371/journal.pbio.0030079

[33] G. Rizzolati, L. Fogassi and V. Gallese, "The Mirror Neuron System: A Motor-Based Mechanism for Action and Intention Understanding," In: The Cognitive Neuroscience, The MIT Press, Cambridge, 2009, pp. 625-640.

[34] N. Gorgoraptis, et al., "Dynamic Updating of Working 
Memory Resources for Visual Objects," Journal of Neuroscience, Vol. 31, No. 23, 2011, pp. 8502-8511. doi:10.1523/JNEUROSCI.0208-11.2011

[35] S. Takahama, S. Miyauchi and J. Saiki, "Neural Basis for Dynamic Updating of Object Representation in Visual Working Memory," Neuroimage, Vol. 49, No. 4, 2010, pp. 3394-3403. doi:10.1016/j.neuroimage.2009.11.029

[36] X. Li, et al., "Blood Oxygenation Level-Dependent Contrast Response Functions Identify Mechanisms of Covert Attention in Early Visual Areas," Proceedings of the National Academy of Sciences, Vol. 105, No. 16, 2008, pp. 6202-6207. doi:10.1073/pnas.0801390105

[37] Z. L. Lu, et al., "Attention Extracts Signal in External Noise: A Bold fMRI Study," Journal of Cognitive Neuroscience, Vol. 23, No. 5, 2011, pp. 1148-1159. doi:10.1162/jocn.2010.21511

[38] A. G. Leventhal, et al., "Concomitant Sensitivity to Orientation, Direction, and Color of Cells in Layers 2, 3, and 4 of Monkey Striate Cortex," Journal of Neuroscience, Vol. 15, No. 3, 1995, pp. 1808-1818.

[39] E. Blaser, T. Papathomas and Z. Vidnyanszky, "Binding of Motion and Colour Is Early and Automatic," European Journal of Neuroscience, Vol. 21, No. 7, 2005, pp. 20402044. doi:10.1111/j.1460-9568.2005.04032.x

[40] A. O. Holcombe and P. Cavanagh, "Early Binding of Feature Pairs for Visual Perception," Nature Neuroscience, Vol. 4, No. 2, 2001, pp. 127-128. doi:10.1038/83945

[41] K. Seymour, et al., "The Coding of Color, Motion, and Their Conjunction in the Human Visual Cortex," Current Biology, Vol. 19, No. 3, 2009, pp. 177-183. doi:10.1016/j.cub.2008.12.050

[42] O. J. Hulme, L. Whiteley and S. Shipp, "Spatially Distributed Encoding of Covert Attentional Shifts in Human Thalamus," Journal of Neurophysiology, Vol. 104, No. 6, 2010, pp. 3644-3656.

[43] S. Shipp, et al., "Feature Binding in the Feedback Layers of Area V2," Cereb Cortex, Vol. 19, No. 10, 2009, pp. 2230-2239. doi:10.1093/cercor/bhn243

[44] A. Treisman and H. Schmidt, "Illusory Conjunctions in the Perception of Objects," Cognitive Psychology, Vol. 14, No. 1, 1982, pp. 107-141. doi:10.1016/0010-0285(82)90006-8

[45] R. Ward, et al., "Deficits in Spatial Coding and Feature Binding Following Damage to Spatiotopic Maps in the Human Pulvinar," Nature Neuroscience, Vol. 5, No. 2, 2002, pp. 99-100.
[46] G. Wolford and K. H. Shum, "Evidence for Feature Perturbations," Percept Psychophys, Vol. 27, No. 5, 1980, pp. 409-420. doi:10.3758/BF03204459

[47] C. Casanova, "The Visual Functions of the Pulvinar," In: The Visual Neurosciences, MIT Press, Cambridge, 2004.

[48] S. Kastner and M. A. Pinsk, "Visual Attention as a Multilevel Selection Process," Cognitive, Affective, \& Behavioral Neuroscience, Vol. 4, No. 4, 2004, pp. 483-500. doi:10.3758/CABN.4.4.483

[49] M. Corbetta and G. L. Shulman, "Control of Goal-Directed and Stimulus-Driven Attention in the Brain," Nature Reviews Neuroscience, Vol. 3, 2002, pp. 201-215.

[50] J. Hopfinger, M. H. Buonocore and G. R. Mangun, "The Neural Mechanisms of Top-Down Attentional Control," Nature Neuroscience, Vol. 3, 2000, pp. 284-291.

[51] S. Kastner, et al., "Increased Activity in Human Visual Cortex during Directed Attention in the Absence of Visual Stimulation," Neuron, Vol. 22, No. 4, 1999, pp. 751761. doi:10.1016/S0896-6273(00)80734-5

[52] T. Liu, et al., "Cortical Mechanisms of Feature-Based Attentional Control," Cereb Cortex, Vol. 13, 2003, pp. 1334-1343.

[53] T. A. Kelley, et al., "Cortical Mechanisms for Shifting and Holding Visuospatial Attention," Cereb Cortex, Vol. 18, No. 1, 2008, pp. 114-125. doi:10.1093/cercor/bhm036

[54] J. T. Serences and S. Yantis, "Spatially Selective Representations of Voluntary and Stimulus-Driven Attentional Priority in Human Occipital, Parietal, and Frontal Cortex," Cereb Cortex, Vol. 17, No. 2, 2007, pp. 284-293. doi:10.1093/cercor/bhj146

[55] R. Vandenberghe, et al., "Functional Specificity of Superior Parietal Mediation of Spatial Shifting," Neuroimage, Vol. 14, 2001, pp. 661-673.

[56] L. Huang, A. Treisman and H. Pashler, "Characterizing the Limits of Human Visual Awareness," Science, Vol. 317, No. 5839, 2007, pp. 823-825. doi:10.1126/science. 1143515

[57] L. Huang and H. Pashler, "A Boolean Map Theory of Visual Attention," Psychological Review, Vol. 114, 2007, pp. 599-631.

[58] L. Huang, "What Is the Unit of Visual Attention? Object for Selection, but Boolean Map for Access," Journal of Experimental Psychology: General, Vol. 139, 2010, pp. 162-179. 